\title{
Guidelines for Weighting Factors Adjustment in Finite State Model Predictive Control of Power Converters and Drives
}

\author{
Patricio Cortés, Samir Kouro, Bruno La Rocca, \\ René Vargas and José Rodríguez \\ Electronics Engineering Department \\ Universidad Técnica Federico Santa María \\ Valparaiso, Chile \\ Email: samir.kouro@ieee.org
}

\author{
José I. León, Sergio Vazquez \\ and Leopoldo G. Franquelo \\ Electronics Engineering Department \\ University of Seville \\ Seville, Spain \\ Email:jileon@zipi.us.es
}

\begin{abstract}
Finite State Model Predictive Control (FS-MPC) has emerged as a promising control tool for power converters and drives. One of the major advantages is the possibility to control several system variables with a single control law, by including them with appropriate weighting factors. However, at the present state of the art, these coefficients are determined empirically. There is no analytical or numerical method proposed yet to obtain an optimal solution. In addition, the empirical method is not always straightforward, and no procedures have been reported. This paper presents a first approach to a set of guidelines that reduce the uncertainty of this process. First a classification of different types of cost functions and weighting factors is presented. Then the different steps of the empirical process are explained. Finally, results for several power converters and drives applications are presented, which show the effectiveness of the proposed guidelines to reach appropriate weighting factors.
\end{abstract}

\section{INTRODUCTION}

The continuous evolution and growing capabilities of modern microprocessors and signal processing technologies, has enabled the implementation of more sophisticated control methods devised to fulfill the industry's increasing demand for higher performance. Predictive control is one of these methods, and has gained recently more attention specially for power converter and drive applications [1]. In essence predictive control is a group of different control methods that share one common characteristic, which is, the use of mathematical models of the system to predict future behaviors and select appropriate control actions. Several predictive control methods have been applied to power converter and drive systems, among them: Dead Beat Control [2]-[8], Model Predictive Control (MPC) [9], [10], Generalized Predictive Control [11], and Finite State Model Predictive Control (FS-MPC) [12].

FS-MPC can be described as a particular case of MPC which takes into account the inherent discrete nature of the power converter switching states and the digital implementation. Since power converters have a finite number of switching states, the MPC optimization problem can be simplified and reduced to the prediction of the system behavior only for those possible switching states. The finite number of system predictions are used to evaluate a cost function (also known as quality or decision function), which usually is composed by the errors of the controlled variables. Hence, the switching state associated with the prediction that minimizes the cost function is selected and generated by the converter. With this approach the number of calculations is greatly reduced, making realtime implementations feasible with current microprocessor technology. FS-MPC has been successfully applied to a wide range of power converters and drives applications [12]-[28].

One of the major advantages of FS-MPC is that several control targets, variables and constraints can be included in a single cost function and simultaneously be controlled. In this way traditional variables such as current, voltage, torque or flux can be controlled while achieving additional control requirements like switching frequency reduction, common mode voltage reduction and reactive power control, to name a few. This can be accomplished simply by introducing the additional control targets in the cost function to be evaluated for the different switching states. However, the combination of variables that most likely are of different nature (different units and different orders of magnitude) in a single cost function is not a straightforward task. Each additional term in the cost function has a corresponding weighting factor, which is used to tune the importance or cost of that term in relation to the others control targets. These parameters have to be properly designed in order to achieve the desired performance. Unfortunately, there are no analytical or numerical methods or control design theories to adjust these parameters, and currently they are determined based on empirical procedures. Although this challenge has not kept back FS-MPC to be applied successfully to several power converters, it is highly desirable to establish a procedure or define some basic guidelines to reduce the uncertainty and improve the effectiveness of the tuning stage.

This paper presents a first approach to address this challenge. First some representative examples of FS-MPC cost functions are classified according to the nature of their terms, in order to group types of weighting factors that 
could be tuned similarly. Then a set of simple guidelines are analyzed and tested to evaluate the evolution of the system performance in relation to changes in the weighting factors. Several converter and drive control applications will be studied to cover a wide variety of cost functions and weighting factors. In addition, results for three different weighting factors are presented to compare results and validate the methodology.

\section{Finite STATE MODEL PREDICTIVE CONTROL OVERVIEW.}

Consider the generic and simplified block diagram of FSMPC illustrated in Fig. 1, that controls a system variable $x$ through a control action $S$, usually the gating signals of a converter. The measured variable $x\left(t_{k}\right)$ is fed back and used to evaluate a discrete predictive model or function of the system $f_{p}$, to obtain the predicted future values of the system $x_{i}^{p}\left(t_{k+1}\right)$ for each possible control action $S_{i}$

$$
x_{i}^{p}\left(t_{k+1}\right)=f_{p}\left\{x\left(t_{k}\right), S_{i}\right\} \quad \forall i=1, \ldots, n .
$$

Note that $n$ corresponds to a finite number of control actions or switching states. Then the $n$ predictions together with the reference are evaluated in a cost function $f_{g}$, leading to $n$ different costs $g$

$$
g_{i}=f_{g}\left\{x^{*}, x_{i}^{p}\right\} \quad \forall i=1, \ldots, n .
$$

Since the target is to control variable $x$, usually the cost function $f_{g}$ is defined by a measure of the error with respect to the reference. Some example of generic cost functions are the absolute error, quadratic error and mean value of the error

$$
\begin{aligned}
g_{i} & =\left|x^{*}-x_{i}^{p}\left(S_{i}\right)\right|, \\
g_{i} & =\left[x^{*}-x_{i}^{p}\left(S_{i}\right)\right]^{2}, \\
g_{i} & =\frac{1}{T_{s}} \int^{T_{S}}\left[x^{*}(t)-x_{i}^{p}\left(t, S_{i}\right)\right] d t .
\end{aligned}
$$

Note that the next control action $S\left(t_{k+1}\right)$ will be the switching state that minimizes the cost function $f_{g}$

$$
S\left(t_{k+1}\right)=\min _{S_{i}} f_{g}\left\{x^{*}, x_{i}^{p}\left(S_{i}\right)\right\} \quad \forall i=1, \ldots, n .
$$

It is clear that FS-MPC takes advantage of the discrete nature of power converters by relating the switching state

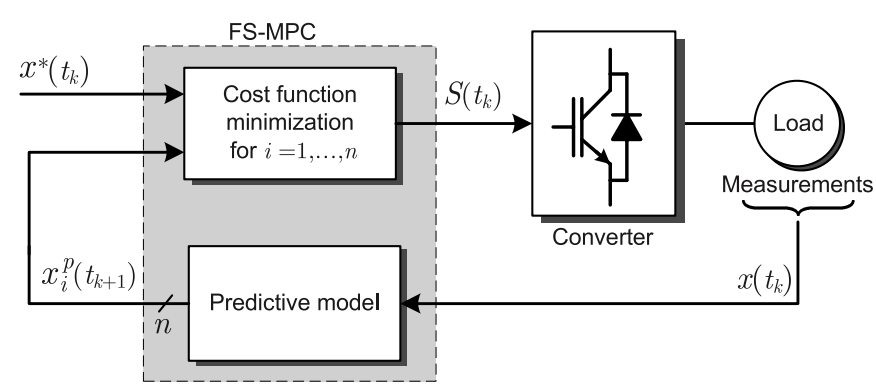

Fig. 1. FS-MPC generic simplified control diagram. directly to the control error. In addition, since the switching state is directly chosen from the cost function minimization, no linear controllers and modulators are necessary. This control principle has been successfully applied to several power converter and drive control systems, including: voltage source inverters, multilevel inverters, matrix converters, regenerative rectifiers and torque control of ac motors, to name a few [11]-[28].

\section{COST FUNCTION CLASSIFICATION.}

Although the cost function's main objective is to keep track of a particular variable and control the system, it is not limited to only do so. In fact one of the main advantages of FS-MPC is that the cost function admits any necessary term that could represent a prediction for another system variable, system constraint or system requirement. This flexibility enables FSMPC to achieve easier more control targets that can translate to increased system performance, efficiency, power quality, safety and other possible figures of merit. Since these terms most likely can be of different physical nature (current, voltage, reactive power, switching losses, torque, flux, etc.) it can lead to coupling effects between variables, or to overestimate the importance of one term respect the others in the cost function, making their presence not worth, hence not controllable.

As mentioned before this issue has been commonly dealt with in MPC by including weighting coefficients or weighting factors $\lambda$, for each term of the cost function

$$
g=\lambda_{x}\left|x^{*}-x^{p}\right|+\lambda_{y}\left|y^{*}-y^{p}\right|+\ldots+\lambda_{z}\left|z^{*}-z^{p}\right| .
$$

Depending on the nature of the different terms involved in the formulation of the cost function, they can be classified in different groups. This classification is necessary in order to facilitate the definition of a weighting factor adjustment procedure that could be applied to similar types of cost functions or alike terms.

\section{A. Cost functions without weighting factors.}

In these kind of cost functions, only one, or the components of one variable, are controlled. This is the simplest case, and since only one type of variable is controlled, no weighting factors are necessary. Some representative examples of this type of cost functions are obtained for: predictive current control of a voltage source inverter [14], predictive power control of a back to back ac/dc/ac converter [16], predictive voltage control of an UPS system [24] and predictive current control with imposed switching frequency [18], among others. The corresponding cost functions are summarized in Table I.

TABLE I

COST FUNCTIONS WITHOUT WEIGHTING FACTORS

\begin{tabular}{cc}
\hline \hline Application & Cost function \\
\hline Current control of a VSI & $\left|i_{\alpha}^{*}-i_{\alpha}^{p}\right|+\left|i_{\beta}^{*}-i_{\beta}^{p}\right|$ \\
Power control of ac/dc/ac converter & $\left|Q^{p}\right|+\left|P^{*}-P^{p}\right|$ \\
Voltage control of UPS & $\left(v_{c \alpha}^{*}-v_{c \alpha}^{p}\right)^{2}+\left(v_{c \beta}^{*}-v_{c \beta}^{p}\right)^{2}$ \\
Imposed switching frequency in a VSI & $\left|F\left(i_{\alpha}^{*}-i_{\alpha}^{p}\right)\right|+\left|F\left(i_{\beta}^{*}-i_{\beta}^{p}\right)\right|$ \\
\hline \hline
\end{tabular}


TABLE III

COST FUNCTIONS WITH EQUALLY IMPORTANT TERMS.

Note that all the terms in a cost functions are composed of variables of the same nature (same unit and order of magnitude). Moreover, some are a decomposition of a single variable into two components. Therefore, no weighting factors and their corresponding tuning are necessary.

\section{B. Cost functions with secondary terms.}

Some systems have a primary goal or a more important control objective that must be achieved in order to provide a proper system behavior, and additional secondary constraints or requirements that should also be accomplished to improve system performance, efficiency or power quality. In this cases the cost function presents a primary and secondary terms, where the importance of the secondary term can vary within a wide range, depending on the application and its specific needs. Some examples are: predictive current control with reduction of the switching frequency to improve efficiency [26], predictive current control with reduction of common mode voltages to prevent motor damage [28], and predictive current control with reactive power reduction to improve power quality [15], [27]. The corresponding cost functions are listed in Table II.

The importance of the second term, i.e. how much the switching frequency, the common mode voltage or the reactive power are reduced, will depend on the specific needs of the application and will impose a tradeoff with the primary control objective, in this case current control. Note that in each cost function a weighting factor $\lambda$ is included with the corresponding secondary term. Hence, solving the tradeoff can be seen as the weighting factor adjustment in the cost function.

\section{Cost functions with equally important terms.}

Unlike the previous case, there are systems in which several variables need to be controlled simultaneously with equal importance in order to control the system. Here the cost function can include several terms with equal importance, and it is the job of the weighting factors to compensate the difference in nature of the variables. Such is the case torque and flux control of an induction machine, where both variables need to be controlled accurately in order to have proper system performance [17]. An other example is current control of an neutral point clamped inverter, in which the dc-link capacitor voltage balance is a must in order to reduce voltage distortion and avoid system damage (exceed the permitted voltage level of the capacitors, otherwise overrated capacitors should be used) [26]. Both cost functions are included in Table III.

TABLE II

COST FUNCTIONS WITH SECONDARY TERMS.

\begin{tabular}{cc}
\hline \hline Application & Cost function \\
\hline Switching frequency reduction & $\left|i_{\alpha}^{*}-i_{\alpha}^{p}\right|+\left|i_{\beta}^{*}-i_{\beta}^{p}\right|+\lambda_{s w} n_{s w}^{p}$ \\
Common mode voltage reduction & $\left|i_{\alpha}^{*}-i_{\alpha}^{p}\right|+\left|i_{\beta}^{*}-i_{\beta}^{p}\right|+\lambda_{c m} V_{c m}^{p}$ \\
Reactive power reduction & $\left|i_{\alpha}^{*}-i_{\alpha}^{p}\right|+\left|i_{\beta}^{*}-i_{\beta}^{p}\right|+\lambda_{Q}\left|Q^{p}\right|$ \\
\hline \hline
\end{tabular}

\begin{tabular}{cc}
\hline \hline Application & Cost function \\
\hline Torque and flux control & $\frac{1}{T_{e n}^{2}}\left(T_{e}^{*}-T_{e}^{p}\right)^{2}+\frac{\lambda_{\psi}}{\psi_{s n}^{2}}\left(\left|\boldsymbol{\psi}_{s}\right|^{*}-\left|\boldsymbol{\psi}_{s}^{p}\right|\right)^{2}$ \\
Capacitor voltage balance & $\frac{1}{i_{s n}}\left[\left|i_{\alpha}^{*}-i_{\alpha}^{p}\right|+\left|i_{\beta}^{*}-i_{\beta}^{p}\right|\right]+\frac{\lambda_{\Delta V}}{V_{c n}}\left|\Delta V_{c}^{p}\right|$ \\
\hline \hline
\end{tabular}

\section{WEIGHTING FACTOR ADJUSTMENT.}

The weighting factor tuning procedure will vary depending on which type of terms are present in the cost function as classified in the previous section.

\section{A. For cost functions with secondary terms.}

This is the easiest case for weighting factor adjustment, since the system can be first controlled using only the primary control objective or term. This can be very simply achieved be neglecting the secondary terms forcing the weighting factor to zero $\lambda=0$. Hence the first step of the procedure is to convert the cost function with secondary terms into a cost function without weighting factors. This will set the starting point for the measurement of the behavior of the primary variable.

The second step is to establish measurements or figures of merit that will be used to evaluate the quality achieved by the weighting factor. For all the examples given in Table II a straightforward quantity should be one related to the primary variable, which is current error. Several error measures for current can be defined, in this work the root mean square (RMS) value of the error in steady state has been used. At least one additional measure is necessary to establish the tradeoff with the secondary term. For the three cost functions of Table II the corresponding measures that were selected are: the device average switching frequency $f_{s w}$, the RMS common mode voltage and the steady state input reactive power.

Once the measures are defined, evaluate the system behavior with simulations starting with $\lambda=0$ and increase the value gradually. Record the corresponding measures for each value of $\lambda$. Stop the increments of $\lambda$ once the measured value for the secondary term has reached the desired value for the specific application, or keep increasing $\lambda$ until the primary variable is not controlled properly. Then plot the results and select a value of $\lambda$ that fulfills the system requirements for both variables.

1) Results for switching frequency reduction: The results of the previous procedure for the first cost function of Table II are given in Fig. 2(a). Here the secondary term is aimed to reduce the switching frequency in a current control of a NPC converter application [26]. The secondary term $n_{s w}^{p}$ corresponds to the predicted number of switchings involved when changing from the present to the future switching state. Thus by increasing the associated weighting factor $\lambda_{s w}$ it is expected that this term gains more importance in the cost function and forces a reduction in the switching frequency, effect that can be clearly observed in Fig. 2(a). However, a reduction in the switching frequency introduces higher distortion affecting the quality of the load current. This tradeoff is very clear in Fig. 2(a) since the curves representing each 


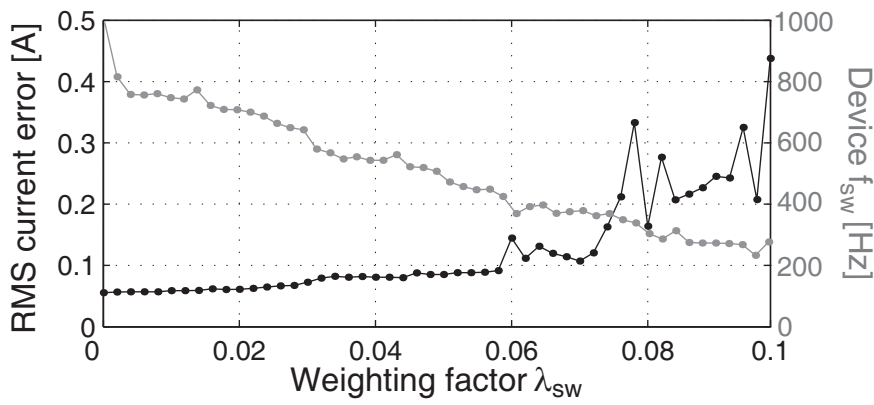

(a)
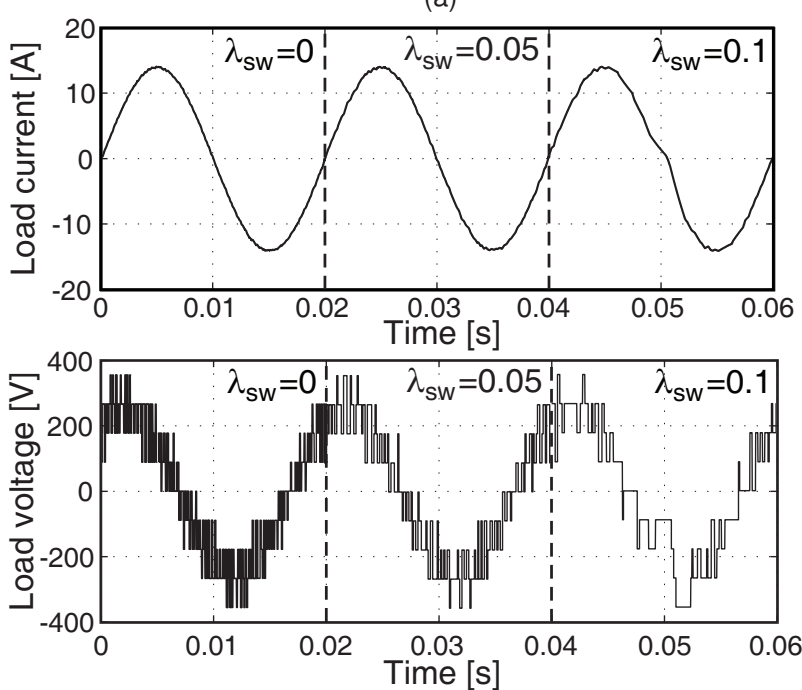

(b)

Fig. 2. a) Weighting factor influence over the current error and the device average switching frequency $f_{s w}$. b) Results comparison for different weighting factors (load current and load voltage).

measure have opposite evolutions for the different values of $\lambda_{s w}$. A suitable selection of $\lambda_{s w}$ would be any value $0.04 \leq \lambda_{s w} \leq 0.06$ since the current error is still below $10 \%$ of the nominal current (15[A] in this example) and a reduction from $1000[\mathrm{~Hz}]$ to $500[\mathrm{~Hz}]$ is achieved for the average device switching frequency. Finally $\lambda_{s w}=0.05$ has been selected. Figure 2(b) shows comparative results for the system working with three different values of $\lambda_{s w}$, one of them the selected value. Note how the load current presents higher distortion for the larger value of $\lambda_{s w}$ due to the strong reduction of the number of commutations. On the other hand for $\lambda_{s w}=0$ the current control works at its best, however at expense of higher switching losses. Since the NPC is aimed for medium voltage high power applications where losses become important, the selection of $\lambda_{s w}=0.05$ merges efficiency with performance.

2) Results for common mode voltage reduction: The guidelines have been used to tune the weighting factor of the second equation of Table II, which corresponds to predictive current control of a matrix converter [28]. Here the additional term $V_{c m}^{p}$ is the predicted common mode voltage for the different switching states and it will be considered an additional cost by tuning properly the weighting factor $\lambda_{c m}$. The measures

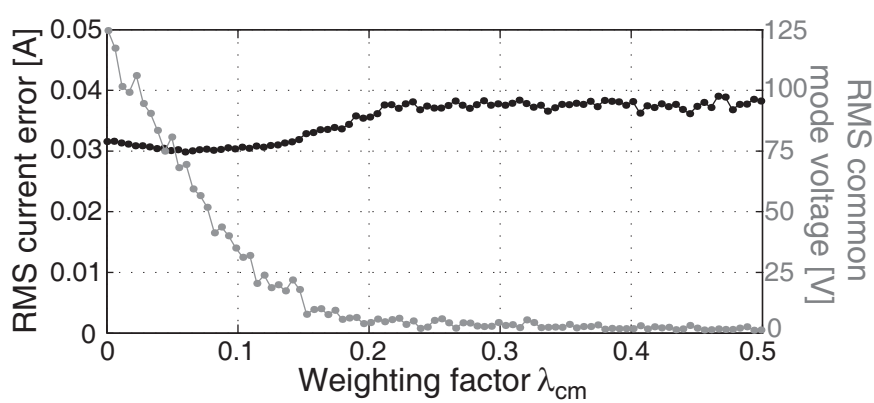

(a)
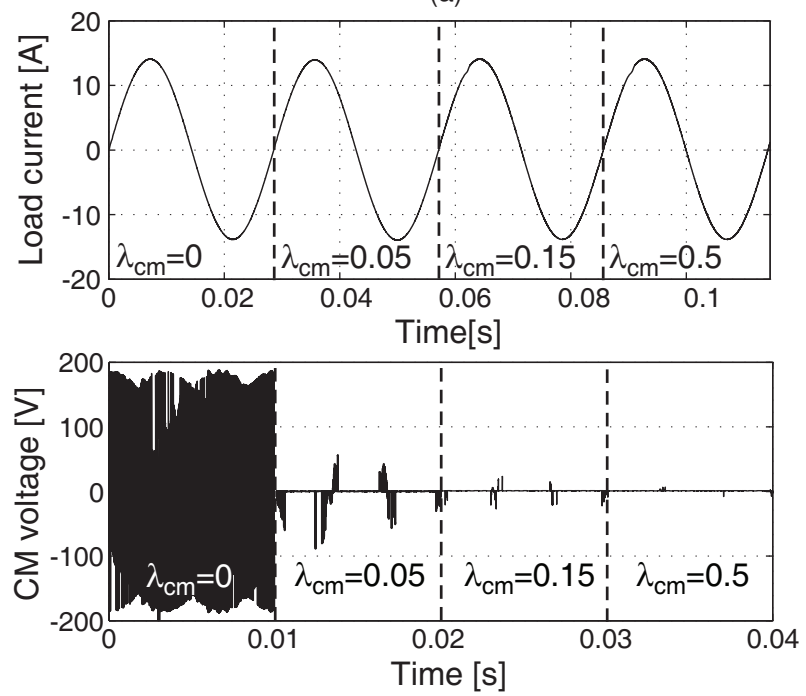

(b)

Fig. 3. a) Weighting factor influence over the current error and the common mode voltage. b) Results comparison for different weighting factors (load current and common mode voltage).

that will be used to evaluate the different $\lambda_{c m}$ are the RMS current error and the RMS common mode voltage. Figure 3(a) shows the results obtained following the proposed procedure. Note that, like for the previous case, similar evolutions of both measures are obtained, i.e, for higher values of $\lambda_{c m}$ smaller $\mathrm{CM}$ voltage are obtained, while the current control becomes less important and looses some performance. The result shows also that CM voltages is a variable more decoupled of the load current compared to the switching frequency since the current error remains very low throughout the wide range of $\lambda_{c m}$. Hence the selection of a appropriate value is easier, and values of $\lambda_{c m} \geq 0.05$ will perform well. This can be observed for the results shown in Fig. 3, where clearly a notorious reduction of the CM voltages is achieved without affecting the current control.

3) Results for input reactive power reduction: The last cost function of Table II corresponds to a current control of a matrix converter [27] with input power factor correction. The additional term in the cost function is directly the predicted input reactive power $Q_{p}$ with its corresponding weighting factor $\lambda_{Q}$. The measures used to tune $\lambda_{Q}$ are the RMS current error and the input reactive power. 


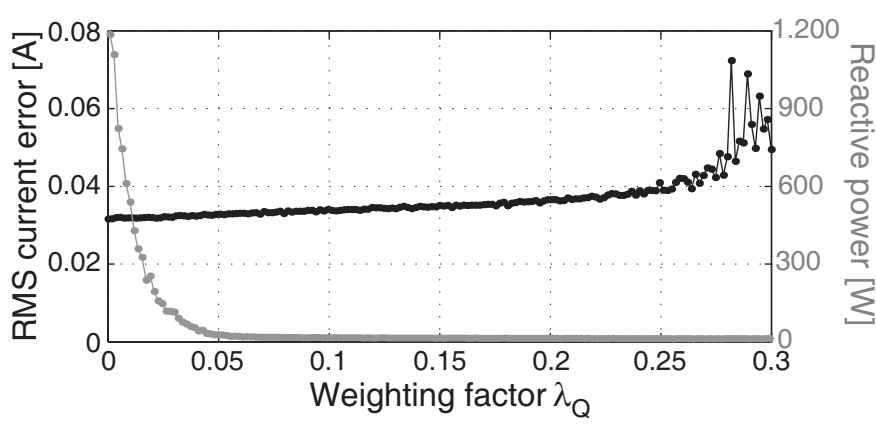

(a)

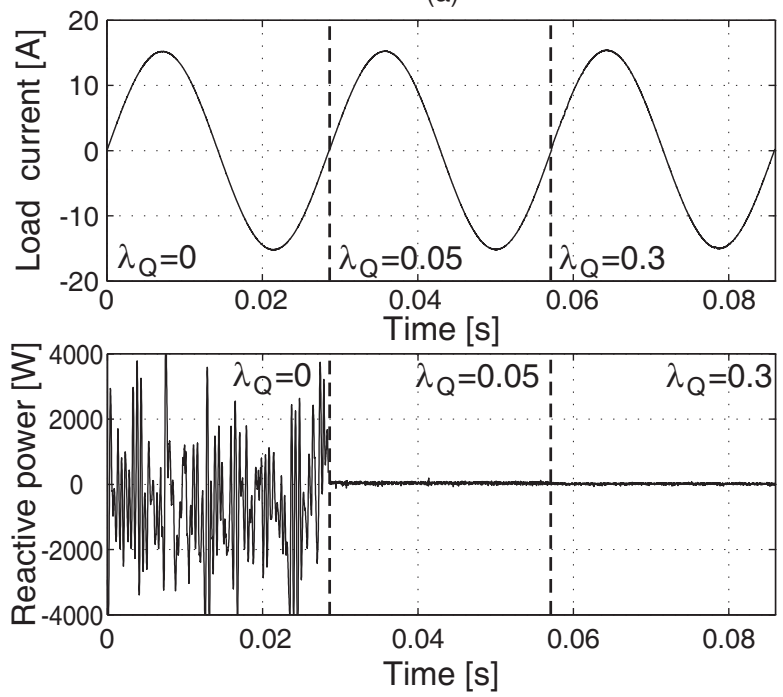

(b)

Fig. 4. a) Weighting factor influence over the current error and the input reactive power. b) Results comparison for different weighting factors (load current and input reactive power).

The results of the tuning procedure are depicted in Fig. 4(a). Since this cost function belongs to the same classification as the previous two, it is expected to present similar measurements evolution when increasing $\lambda_{Q}$. As with the previous case, the input reactive power seems to be very decoupled of the load current, hence the current error remains very low for a wide range of $\lambda_{Q}$. It becomes easy to obtain a suitable value considering $\lambda_{Q} \geq 0.05$. This can be corroborated with the results given in Fig. 4(b), showing an important reduction of the input reactive power for $\lambda_{Q}=0.05$.

The proposed procedure can be programmed by automating and repeating the simulation introducing an increment in the weighting factor after each simulation. An other way is to reduce the number of repetitions, by applying a branch and bound algorithm. For this approach first select a couple of initial values for $\lambda$, usually with different orders of magnitude to cover a very wide range $\lambda=0,0.1,1$ and 10 , for example. A qualitative example of this algorithm is illustrated in Fig. 5. Then simulate for these weighting factors and obtain the measures for both terms, $M^{1}$ and $M^{2}$ for the primary and secondary terms respectively. Then compare these results with the desired maximum errors admitted by the application and fit them into an interval of two weighting factors $(0.1 \leq \lambda \leq 1$

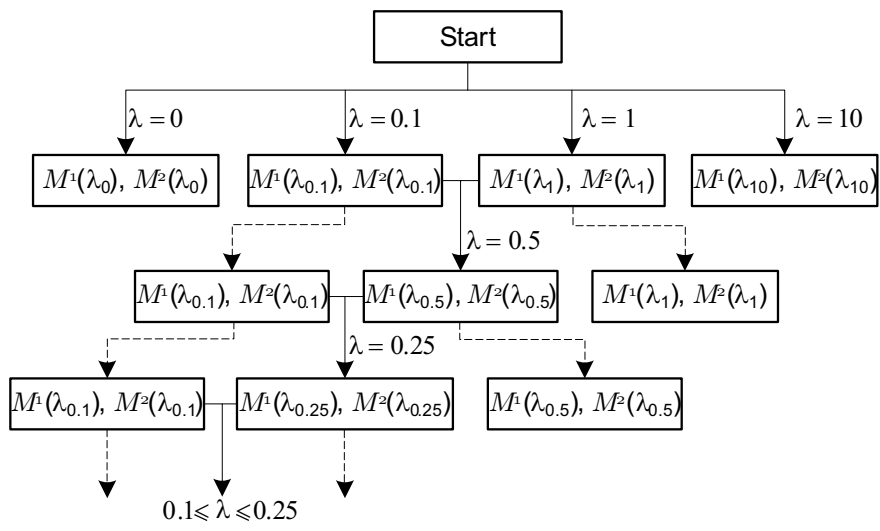

Fig. 5. Branch and bound algorithm to reduce simulations to obtain suitable weighting factors.

in the example). Then compute the measures for the $\lambda$ in the half of the new interval ( $\lambda=0.5$ in the example) and continue so on until you achieve a suitable lambda. Note in Fig. 5 that each hard line corresponds to a simulation and dashed lines corresponds to values already simulated. This method reduces the number of simulations necessary to obtain a working weighting factor.

The qualitative example of Fig. 5 can be matched with the results for the common mode reduction case of Fig. 3(a). Note that with only 7 simulations the search for $\lambda_{c m}$ would have narrowed to an interval $0.1 \leq \lambda_{c m} \leq 0.25$ where any $\lambda_{c m}$ would work properly.

\section{B. For cost functions with equally important terms}

For cost functions like those listed in Table III, the procedure needs some minor adjustments since $\lambda$ is not allowed to be zero. Another difficulty is the different nature of the variables. For example, when controlling torque and flux in an adjustable speed drive application with a nominal torque and flux of $25[\mathrm{Nm}]$ and $1[\mathrm{~Wb}]$ respectively, the torque error can have different orders of magnitudes making both variable not equally important in the cost function, affecting the system performance. Thus the fist step is to normalize the cost function. Once normalized, all the terms will be equally important and now $\lambda=1$ can be considered as starting point. Usually a suitable $\lambda$ is closely located to 1 . Note that the cost functions in Table III have already included this normalization (nominal values are denoted by subindex $n$ ).

The second step is the same as with the previous procedure, i.e., measurements or figures of merit have to be defined that will be used to evaluate the quality achieved by the weighting factor.

The last step is to perform the branch and bound algorithm of Fig. 5 considering a couple of starting points. Naturally $\lambda=1$ has to be considered, and $\lambda=0$ has to be avoided. When a small interval of weighting factors has been reached, meaning by small interval, that there are no big differences in the measures between the upper and lower bounds of the interval, then the weighting factor has been obtained. 


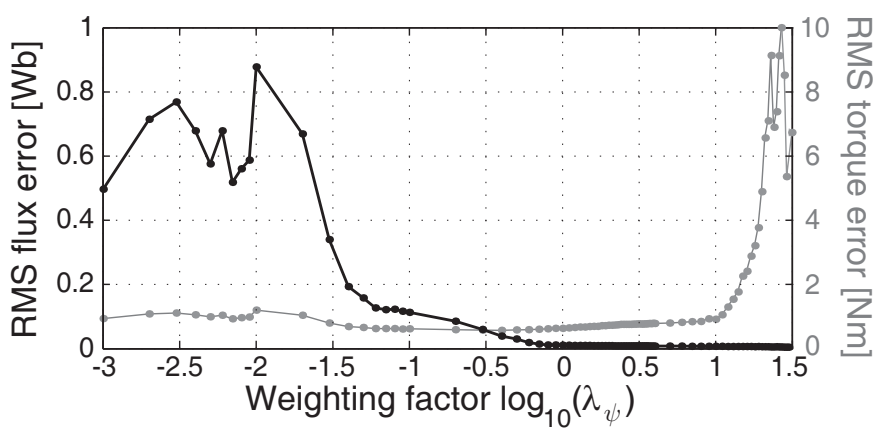

(a)
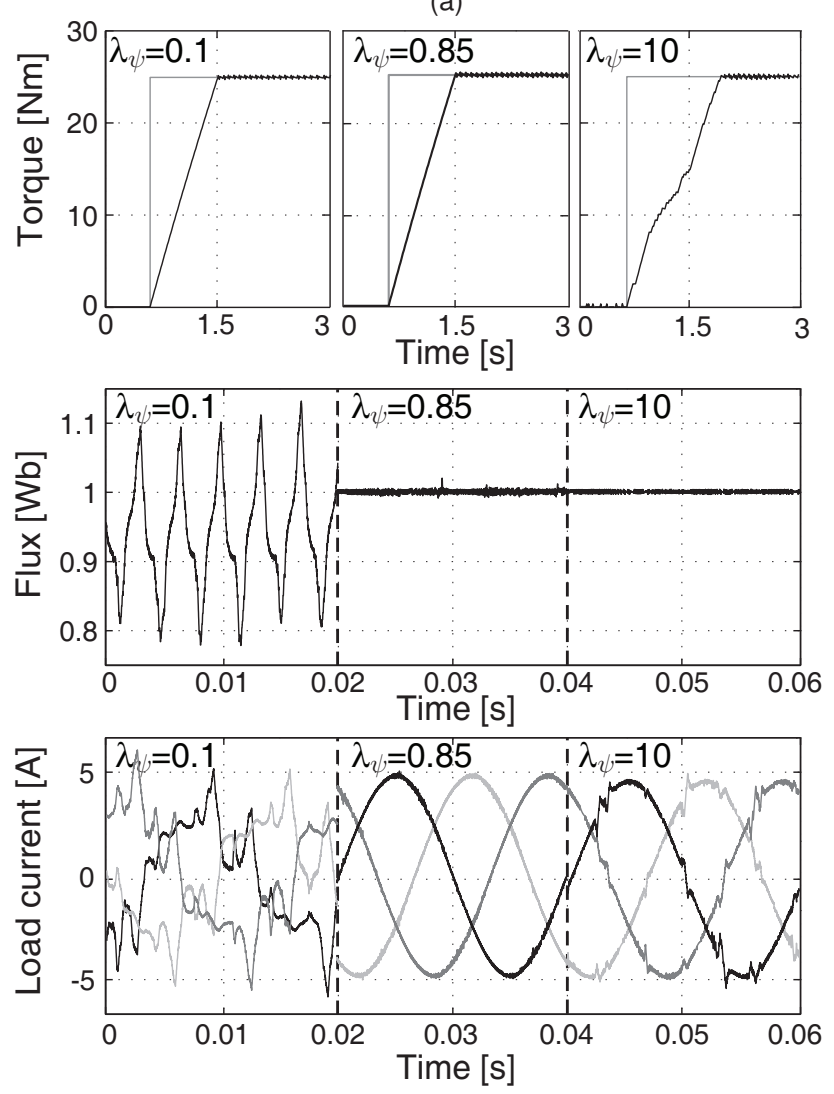

(b)

Fig. 6. a) Weighting factor influence over the flux and torque errors. b) Results comparison for different weighting factors (torque step response, flux magnitude in steady state and load currents).

1) Results for torque and flux control: The predictive torque and flux control of an induction motor speed drive [17], can be implemented using the first cost function in Table III. Note that the terms appear already normalized. The two terms in the cost function are the torque and flux error. Hence the measures that will be suitable to select the proper $\lambda_{\psi}$ will be the respective RMS errors. A branch and bound algorithm starting with $\lambda_{\psi}=0.01,0.1,1,10$ and 100 first gave the interval $0.1 \leq \lambda_{\psi} \leq 1$, and then $0.5 \leq \lambda_{\psi} \leq 1$ with very small differences. Finally $\lambda_{\psi}=0.85$ was chosen. Figure 6(a) shows extensive results considering much more values of $\lambda_{\psi}$ (note that the values are represented in $\log _{10}()$ scale), to show that the branch and bound method really found a suitable solution.

Results for different $\lambda_{\psi}$, including $\lambda_{\psi}=0.85$ are given in Fig. 6(b) to show the performance achieved by the FS-MPC.

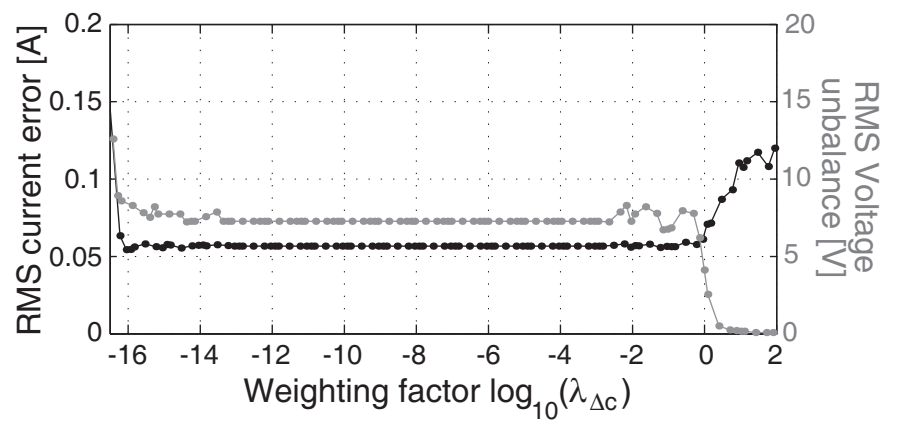

(a)
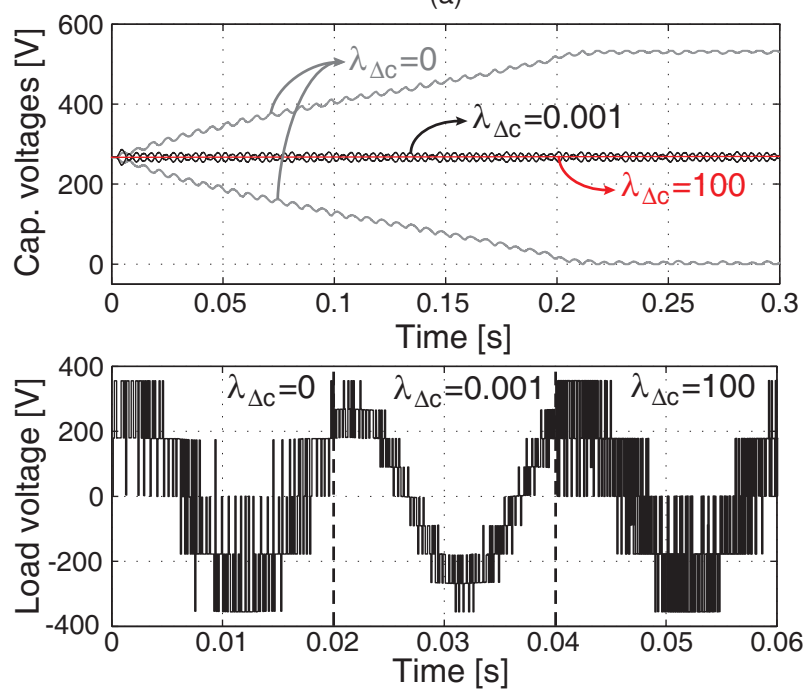

(b)

Fig. 7. a) Weighting factor influence over the current error and the dc-link capacitors unbalance. b) Results comparison for different weighting factors (load current and dc-link capacitor voltages dynamic behavior).

Note that $\lambda_{\psi}=0.85$ presents the best combination of torque step response and steady state, flux control and load current waveforms.

2) Results for voltage balancing: The predictive current control of a NPC converter [26], can be implemented using the second cost function in Table III. The additional term $\Delta V_{c}^{p}$ corresponds to the predicted voltage unbalance of the dc-link capacitors of the converter. If this unbalance is not controlled, the dc-link voltages will drift and introduce considerable output voltage distortion, no to mention that the dc-link capacitors could get damaged by overvoltage, unless they are overrated. Note the terms appear already normalized in the cost function, as indicated in the first step of the procedure. The measures that will be used to evaluate the weighting factor $\lambda_{\Delta V}$ are the RMS current error and the peak amplitude of the voltage unbalance.

A branch and bound algorithm starting with $\lambda_{\Delta V}=10^{-4}$, $10^{-2}, 1,10^{2}$ and $10^{4}$ first gave the interval $10^{-4} \leq \lambda_{\Delta V} \leq$ $10^{-2}$ after this first evaluation very small differences were obtained. Finally $\lambda_{\Delta V}=10^{-3}$ was evaluated leading to the same measures. Hence this value was chosen. Figure 7(a) shows extensive results considering much more values (note that the values are represented in $\log _{10}()$ scale), to show that the branch and bound method really found a suitable solution. 
Results for different $\lambda_{\psi}$, including $\lambda_{\Delta V}=0.001$ are given in Fig. 7(b) to show the performance achieved by the FS-MPC. Note that for $\lambda_{\Delta V}=0$, which normally is not allowed since its does not control the unbalance producing the maximum drift of the dc-link capacitors, the load voltage only presents 5 different voltage levels, while 9 levels should appear in the load phase-neutral voltage (since the NPC has 3 levels in the converter phase-neutral voltage). Only 5 appear since the NPC is not generating 3 output levels, due to the voltage drift of its capacitors it is only generating 2 levels. On the other hand, $\lambda_{\Delta V}=100$ controls the voltage unbalance very accurately, it even makes voltage unbalance so important in the cost function that it disables the generation of those switching states that produce unbalance eliminating voltage levels at the output and increases the switching frequency as can be seen in the load voltage of Fig. 7(b). On the contrary, the selected $\lambda_{\Delta V}=0.001$ presents the 9 load voltage levels, controls the load current and keeps the capacitor voltages balanced.

\section{CONCLusion}

In this paper the design of the weighting factors used in cost functions of Finite State Model Predictive Control has been analyzed. A first approach based on an empirical procedure to obtain suitable weighting factors has been presented.

For cost functions with a primary control objective and secondary terms, the starting point is $\lambda=0$, then test increments of $\lambda$ until the desired behavior is obtained (branch and bound can also be used). For cost functions with equally important terms, first normalize the cost function and set $\lambda=1$, with this value the system will be controlled, for fine tuning use branch and bound or move slightly $\lambda$ around 1 . At least two different figures of merit or system parameters have to be considered, depending on the application, to settle the tradeoff present in the designing choice of the weighting factors.

This contribution is a first design approach to reduce the uncertainty if the cots function design in FS-MPC of systems with more than one control objective. The examples studied in this paper show the potential and flexibility of FS-MPC and how easy it is to include additional control objectives in one single controller compared to classic control schemes.

\section{REFERENCES}

[1] R. Kennel and A. Linder, "Predictive control of inverter supplied electrica drives," IEEE Power Electronics Specialists Conference (PESC 2000), pp. 761-766, Galway, Ireland, 2000.

[2] O. Kukrer, "Discrete-time current control of voltage-fed three-phase PWM inverters," IEEE Trans. on Industrial Electronics, vol. 11, no. 2, pp. 260-269, March 1996.

[3] H.-T. Moon, H.-S. Kim, and M.-J. Youn, "A discrete-time predictive current control for PMSM," IEEE Trans. On Power Electronics, vol. 18, no. 1, pp. 464-472, January 2003.

[4] L. Springob and J. Holtz, "High-bandwidth current control for torqueripple compensation in PM synchronous machines," IEEE Trans. On Industrial Electronics, vol. 45, no. 5, pp. 713-721, October 1998.

[5] G. Bode, P. C. Loh, M. J. Newman, and D. G. Holmes, "An improved robust predictive current regulation algorithm," IEEE Trans. on Industry Applications, vol. 41, no. 6, pp. 1720-1733, November 2005.

[6] S.-M. Yang and C.-H. Lee, "A deadbeat current controller for field oriented induction motor drives," IEEE Trans. on Power Electronics, vol. 17, no. 5, pp. 772-778, September 2002.
[7] H. Abu-Rub, J. Guzinski, Z. Krzeminski, and H. A. Toliyat, "Predictive current control of voltage source inverters," IEEE Transactions on Industrial Electronics, vol. 51, no. 3, pp. 585-593, June 2004.

[8] P. Mattavelli, "An improved deadbeat control for UPS using disturbance observers," Trans. on Industrial Electronics, vol. 52, no. 1, pp. 206-212, Feb. 2005.

[9] E. F. Camacho and C. Bordons, "Model Predictive Control," SpringerVerlag, 1999.

[10] A. Linder and R. Kennel, "Model predictive control for electrical drives," in Proc. of IEEE PESC 05, Recife, Brazil, June 12-16 2005, pp. 17931799.

[11] R. Kennel, A. Linder, and M. Linke, "Generalized predictive control (GPC)-ready for use in drive applications?” IEEE 32nd Annual Power Electronics Specialists Conference, PESC01 , vol. 4, pp. 1839-1844, 2001.

[12] P. Cortes, J. Rodriguez, R. Vargas, and U. Ammann, "Cost functionbased predictive control for power converters," in IEEE Industrial Electronics, IECON 2006 - 32nd Annual Conference on, Nov. 2006, pp. 2268 2273.

[13] S. Muller, U. Ammann, and S. Rees, "New modulation strategy for a matrix converter with a very small mains filter," IEEE 33th Annual Power Electronics Specialists Conference, PESC03, pp. 1275-1280, Acapulco, Mexico, 2003.

[14] J. Rodríguez, J. Pontt, C. Silva, P. Correa, P. Lezana, P. Cortés, and U. Ammann, "Predictive current control of a voltage source inverter," IEEE Trans. on Industrial Electronics, vol. 54, no. 1, pp. 495-503, February 2007.

[15] S. Muller, U. Ammann, and S. Rees, "New time-discrete modulation scheme for matrix converters," IEEE Trans. on Industrial Electronics, vol. 52, no. 6, pp. 1607-1615, December 2005.

[16] J. Rodriguez, J. Pontt, P. Correa, P. Lezana, and P. Cortes, "Predictive power control of an AC/DC/AC converter,' IEEE Industry Applications Society Annual Meeting, IAS'05, vol. 2, pp. 934-939, Oct. 2005.

[17] J. Rodriguez, J. Pontt, C. Silva, P. Cortés, S. Rees, and U. Ammann, "Predictive direct torque control of an induction machine," in 11th International Power Electronics and Motion Control Conference, EPEPEMC 2004, Riga, Latvia, 2-4 September 2004.

[18] P. Cortes, J. Rodriguez, D. E. Quevedo, and C. Silva, "Predictive current control strategy with imposed load current spectrum," IEEE Transactions on Power Electronics, vol. 23, no. 2, pp. 612-618, Mar. 2008.

[19] A. Linder and R. Kennel, "Direct model predictive control - a new direct predictive control strategy for electrical drives," in Power Electronics and Applications, 2005 European Conference on, Sept. 2005.

[20] G. Perantzakis, F. Xepapas, S. Papathanassiou, and S. N. Manias, "A predictive current control technique for three-level NPC voltage source inverters," in Power Electronics Specialists Conference, 2005. PESC '05. IEEE 36th, Sept. 2005, pp. 1241-1246.

[21] G. S. Perantzakis, F. H. Xepapas, and S. N. Manias, "Efficient predictive current control technique for multilevel voltage source inverters," in Power Electronics and Applications, 2005 European Conference on, Sept. 2005.

[22] H. Q. S. Dang, P. Wheeler, and J. Clare, "A control analysis and implementation of high voltage, high frequency direct power converter," in IEEE Industrial Electronics, IECON 2006 - 32nd Annual Conference on, Nov. 2006, pp. 2096-2102.

[23] M. Catucci, J. Clare, and P. Wheeler, "Predictive control strategy for ZCS single stage resonant converter," in IEEE Industrial Electronics, IECON 2006 - 32nd Annual Conference on, Nov. 2006, pp. 2905-2910.

[24] P. Cortes and J. Rodriguez, "Three-phase inverter with output LC filter using predictive control for UPS applications," in Power Electronics and Applications, 2007 European Conference on, Sept. 2007, pp. 1-7.

[25] E. I. Silva, B. P. McGrath, D. E. Quevedo, and G. C. Goodwin, "Predictive control of a flying capacitor converter," in Proceedings of the American Control Conference, New York City, USA, July 2007.

[26] R. Vargas, P. Cortes, U. Ammann, J. Rodriguez, and J. Pontt, "Predictive control of a three-phase neutral-point-clamped inverter," IEEE Transactions on Industrial Electronics, vol. 54, no. 5, pp. 2697-2705, Oct. 2007.

[27] R. Vargas, M. Rivera, J. Rodríguez, J. Espinoza, Predictive Torque Control with Input PF Correction applied to an Induction Machine fed by a Matrix Converter, in Conf. Rec. of IEEE PE Society Annual Meeting, PESC 2008, 15-19 June 2008.

[28] R. Vargas, U. Ammann, J. Rodríguez, J. Pontt, Predictive Strategy to Reduce Common-Mode Voltages on Power Converters, in Power Electronics Specialists Conference, PESC 2008, 15-19 June 2008. 